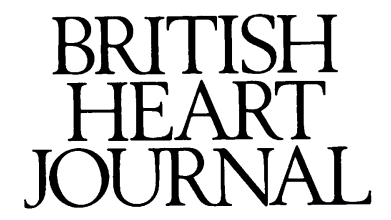

Editorial

\title{
Advances in rate responsive pacing?
}

An exercise related increase in ventricular rate in patients with permanent pacemakers has been clearly shown to improve exercise tolerance in nearly all ambulant patients undergoing pacemaker implantation. ${ }^{1-5}$ There is still controversy about whether the maintenance of atrioventricular synchrony is important, with many studies showing little difference in exercise tolerance or haemodynamic variables with DDD or VVIR pacing. ${ }^{3-5}$ Atrioventricular synchrony is probably more important at rest than during exercise and most patients seem to prefer DDD pacing to VVIR pacing. ${ }^{56}$ The superiority of the normal sinus node over artificial sensors, however, makes DDD pacing a better rate responsive system than VVIR pacing in most patients.

\section{The sinus node as sensor}

The normal sinus node is the ideal physiological sensor but there are problems associated with using atrial sensing to determine ventricular rate. These include abnormal sinus node function, atrial arrhythmias, and the practical aspects of atrial sensing. The sinus node may be abnormal because of chronotropic incompetence or may be denervated in the transplanted heart. Atrial arrhythmias will trigger inappropriate ventricular responses and abolish any useful rate response. An atrial lead is required in patients with atrioventricular nodal disease and some extra expertise and costs are needed for implantation and follow up of DDD pacemakers.

\section{Artificial sensors}

Sensor driven rate responsive pacing is still clearly indicated those patients with moderate or severe chronotropic incompetence or atrial arrhythmias. Patients with the sick sinus syndrome and chronotropic incompetence will still require atrial pacing, either with AAIR or DDDR pacemakers, because this will reduce the incidence of atrial fibrillation, systemic emboli, and left ventricular failure. ${ }^{7}$ Patients with chronic atrial fibrillation are best treated with VVIR pacemakers.

The patient with intermittent atrial arrhythmias may be best treated with a mode switching DDDR pacemaker. These pacemakers will switch to VDIR either by sensing very short atrial intervals or because the output from the artificial sensor suggests that the atrial rate is inappropriate. ${ }^{89}$ This technology can be used for patients who have undergone atrioventricular junctional ablation for intermittent atrial arrhythmias. These patients in the past have been restricted to VVIR pacing, DDIR pacing, or DDD pacing with a low upper rate limit, which has reduced their quality of life when in sinus rhythm. Mode switching DDDR pacing will allow normal atrioventricular synchrony during sinus rhythm and VDIR pacing during tachycardia.

Artificial sensors allow a single or dual chamber rate response but thereare problems with the physiological nature of that response, and different problems with different sensors. ${ }^{6}{ }^{10}$ The most widely used sensor is the piezo-electric crystal, which responds to vibration. This system allows a rapid heart response to most activity, but there are associated difficulties. The response is not easily graded and cardiac output demand and degree of vibration are not always closely related-for example, going up or down stairs. ${ }^{11}$ This system allows no physiological feedback and may require fine tuning. New pacemakers have heart rate Holter facilities that allow reprogramming without the need for formal exercise testing. ${ }^{12}$

\section{The future}

Intensive research efforts are being made to overcome the problems with existing sensors and new sensor systems or multiple sensor systems are being tested. At present there do not seem to be any major advances in sensor technology. ${ }^{10} \mathrm{~A}$ wide range of sensors is being developed and tested, either pacemaker based (thoracic impedance, evoked QT or acceleration) or pacing electrode based (temperature, $\mathrm{dP} / \mathrm{dt}$, right atrial pressure, pulmonary artery pressure, $\mathrm{pH}$, mixed venous saturation, and catecholamine concentrations). The present state of their development is detailed in an excellent review by Lau. ${ }^{10}$ Mixed venous oxygen saturation is the most promising, because it may be the most physiological with a rapid proportional response to exercise and appropriate slowing in recovery..$^{13}$ It is also attractive because it allows a clear negative feedback loop, and will therefore need little programming even if the patient's cardiac function changes. Existing lead technology, however, uses an optical sensor and light emitting diode within the pacemaker electrode. This stops working if it becomes coated with fibrin and thrombrin. It also has a drawback that is common to all electrode based technology-if there is malfunction or a need for an upgrade it may not be possible to remove the old lead.

Dual (or multiple) artificial sensors at present merely seem to be the sum of their imperfect parts, and they increase the size, cost, and current drain of the pacing 
unit. In patients with either chronotropic incompetence or mode switching DDDR pacemakers the best dual sensor system is the normal sinus node and an artificial sensor. ${ }^{8-10}$

So where does this leave sensor driven, rate responsive pacing? There have been no major advances in sensors and therefore operators should continue to use the sensor with which they feel comfortable. Ambulant patients with chronotropic incompetence should have AAIR or DDDR pacemakers, and those with chronic atrial tachycardias should have VVIR pacemakers. In patients with intermittent atrial arrhythmias, the atrium should still be paced and sensed. This will probably reduce the occurrence of chronic atrial fibrillation and systemic emboli, as has been repeatedly shown in the sick sinus syndrome. Ideally in these patients the capabilities of sensor driven rate response and mode switching should be included to optimise pacing during intermittent or chronic atrial arrhythmias. Should patients with normal sinus nodes and atrioventricular block be given DDDR pacemakers with these capabilities? Up to $10 \%$ of these patients with atrioventricular block will go on to chronic atrial fibrillation or have intermittent atrial arrhythmias. ${ }^{14}$ This then becomes a question of cost. If a DDDR pacemaker costs much more than a DDD pacemaker (as it does in the United Kingdom) the answer is no. If the prices of both types are similar (as in the USA) then it is sensible to give the patient all the options with a DDDR pacemaker.

In conclusion, atrial sensing is the best rate responsive system for patients with normal sinus node function. Sensor driven rate response is important for patients with chronotropic incompetence or chronic atrial tachycardias. Patients with intermittent atrial tachycardias may have the best of both worlds from a mode switching DDDR/VDIR pacemaker, and this in conjunction with atrioventricular junctional ablation may be the best treat- ment for patients with drug resistant paroxysmal atrial fibrillation.

Cardiac Department, MICHAEL GRIFFITH

Queen Elizabeth Hospital,Birmingham

1 Sutton R, Perrins EJ, Morley C, Chan SL. Sustained improvement in exercise tolerance following physiological cardiac pacing. Eur Heart $\mathcal{F}$ 1983;4:781-5.

2 McMeekin, JD, Lautner D, Hanson S, Gulamhusein SS. Importance of heart rate response during exercise in patients using atrioventricular synchronous and ventricular pacemakers. PACE 1990;13:59-68.

3 Linde-Edelstam C, Hjemdahl P, Pehrsson SK, Astrom H, Norlander R. Is DDD pacing superior to VVIR? A study on cardiac sympathetic nerve activity and myocardial oxygen consumption at rest and during exercise. activity and myocardial

4 Oldroyd KG, Rae AP, Carter R, Wingate C, Cobbe SM. Double blind crossover comparison of the effects of dual chamber pacing (DDD) and ventricular rate adaptive (VVIR) pacing on neuroendocrine variables, exercise performance, and symptoms in complete heart block. $\mathrm{Br}$ Heart f 1991;65:188-93.

5 Lau CP, Wong CK, Leung WH, Liu WX. Superior cardiac haemodynamics of atrioventricular synchrony over rate responsive pacing at submaximal exercise: observations in activity sensing DDDR pacemakers. PACE 1990;13:1832-7.

6 Sulke AN, Pipilis A, Henderson RA, Bucknall CA, Sowton E. Comparison of the normal sinus node with seven types of rate responsive pacemaker during everyday activity. Br Heart $\mathcal{f}$ 1990;64:25-31.

7 Rosenqvist $M$, Brandt J, Schuller $\mathrm{H}$. Long term pacing in sinus node disease: Effects of stimulation mode on cardiovascular morbity and mortality. Am Heart $\mathcal{f}$ 1988;116:16-22.

8 Lee MT, Adkins A, Woodson D, Vandegriff J. A new feature for control of inappropriate high rate tracking in DDDR pacemakers. PACE 1990;13:1852-5.

9 Lau CP, Tai YT, Fong PC, Li JPS, Chung FLW, Song S. The use of implantable sensors for the control of pacemaker mediated tachycardias: a comparative evaluation between minute ventilation sensing and acceleration sensing dual chamber rate responsive adaptive pacemakers. $P A C E$ 1992;15:34-44.

$10 \mathrm{Lau} C \mathrm{CP}$. The range of sensors and algorithms used in rate adaptive cardiac pacing. $P A C E$ 1992;15:1177-211.

11 Lau CP, Mehta D, Toff WD, Stott RI, Ward DE, Camm AJ. Limitations of rate response of an activity sensing rate-responsive pacemaker to different forms of activity. PACE 1988;11:141-50.

12 Mahaux V, Waleffe A, Kulbertus $H$. Usefulness and adequacy of sensor data storage and retrieval for rate responsive pacing. PACE data storage and

13 Faerestrand S, Ohm O-J. Long term follow up of a rate-variable pacemaker controlled by central venous oxygen saturation [abstr]. $\mathcal{f} \mathrm{Am}$ Coll Cardiol 1991;17:289.

14 Gross J, Moser S, Benedek ZM, Carolyn Andrews, Furman S. Clinical predictors and natural history of atrial fibrillation in patients with DDD pacemakers. PACE 1990;13:1828-31. 